\title{
Nonclassical CD1d-restricted NK T cells that produce IL-13 characterize an atypical Th2 response in ulcerative colitis
}

\author{
Ivan J. Fuss, ${ }^{1}$ Frank Heller, ${ }^{1}$ Monica Boirivant, ${ }^{2}$ Francisco Leon, ${ }^{3}$ Masaru Yoshida, ${ }^{4}$ \\ Stefan Fichtner-Feigl,, ${ }^{1}$ Zhiqiong Yang, ${ }^{1}$ Mark Exley, ${ }^{5}$ Atsushi Kitani, ${ }^{1}$ Richard S. Blumberg, ${ }^{4}$ \\ Peter Mannon, ${ }^{1}$ and Warren Strober ${ }^{1}$
}

\begin{abstract}
${ }^{1}$ Mucosal Immunity Section, NIH, Bethesda Maryland, USA. ${ }^{2}$ Laboratory of Immunology, Istituto Superiore di Sanità, Rome, Italy. ${ }^{3}$ mmune Cell Interaction Unit, NIH, Bethesda, Maryland, USA. ${ }^{4}$ Division of Gastroenterology, Brigham and Women's Hospital, Boston Massachusetts, USA. ${ }^{5}$ Cancer Biology Program, Hematology/Oncology Division, Beth-Israel Deaconess Medical Center, Harvard Medical School, Boston, Massachusetts, USA.
\end{abstract}

\begin{abstract}
While Crohn disease (CD) has been clearly identified as a Th1 inflammation, the immunopathogenesis of its counterpart inflammatory bowel disease, ulcerative colitis (UC), remains enigmatic. Here we show that lamina propria T (LPT) cells from UC patients produce significantly greater amounts of IL-13 (and IL-5) than control cells and little IFN- $\gamma$, whereas comparable cells from CD patients produce large amounts of IFN- $\gamma$ and small amounts of IL-13. We then show that stimulation of UC LPT cells bearing an NK marker (CD161) with anti-CD2/ anti-CD28 or with B cells expressing transfected CD1d induces substantial IL-13 production. While this provided firm evidence that the IL-13-producing cell is an NK T (NKT) cell, it became clear that this cell does not express invariant NKT cell receptors characteristic of most NKT cells since there was no increase in cells binding $\alpha$-galactosylceramide-loaded tetramers, and $\alpha$-galactosylceramide did not induce IL-13 secretion. Finally, we show that both human NKT cell lines as well as UC CD161 ${ }^{+}$LPT cells are cytotoxic for HT-29 epithelial cells and that this cytotoxicity is augmented by IL-13. These studies show that UC is associated with an atypical Th2 response mediated by nonclassical NKT cells producing IL-13 and having cytotoxic potential for epithelial cells.
\end{abstract}

\section{Introduction}

Studies of murine models of the two forms of inflammatory bowel disease (IBD), Crohn disease (CD) and ulcerative colitis (UC), strongly suggest that both of these diseases are due to dysregulation of the mucosal immune response to antigens in the mucosal microflora $(1,2)$. Nevertheless, the fact that CD and UC differ from one another both clinically and pathologically gave rise to the notion fairly early on that very different immune processes are at play in the two diseases. In particular, CD is marked by a transmural, granulomatous inflammatory process that is usually associated with Th1 responses, whereas UC is a more superficial disease in which epithelial damage is an over-riding factor, and autoantibodies are usually present that are generally markers of Th2 responses (3). These differences are also borne out by studies of the immune profiles in CD and UC that show that CD is associated with an IL-12-directed IFN- $\gamma$ response, a hallmark of a Th1 inflammation, whereas in UC this type of response is not present, and some other type of immune response, possibly a Th2 response, is evidently driving the inflammation (4-8).

To gain further insight into the possible role of Th2-driven processes in the inflammation in $\mathrm{UC}$, one can turn to various murine models of inflammation that are dependent on a Th2 response (2). Of the several such models available, one that has proven particular-

Nonstandard abbreviations used: 721.221 cell line (721 cells); concanavalin A (conA); Crohn disease (CD); effector-to-target ratio (E/T); $\alpha$-galactosylceramide $(\alpha$-GalCer); inflammatory bowel disease (IBD); intestinal epithelial cell (IEC); invariant NKT (iNKT); lamina propria mononuclear cell (LPMC); NK T (NKT); phycoerythrin (PE); T cell receptor (TCR); ulcerative colitis (UC).

Conflict of interest: The authors have declared that no conflict of interest exists.

Citation for this article: J. Clin. Invest. 113:1490-1497 (2004).

doi:10.1172/JCI200419836. ly useful is oxazolone colitis, a colitis induced by intrarectal administration of the haptenating agent, oxazolone. As shown initially by Boirivant et al., when oxazolone is administered intrarectally without prior sensitization, one sees a rapidly developing and spontaneously resolving inflammation histologically reminiscent of UC, in that it is marked by a superficial, edematous, ulcerative lesion that affects the distal half of the colon (9). This lesion is a Th2-driven process characterized by the production of IL- 4 and IL- 5 and, indeed, is prevented by the administration of anti-IL- $4 \mathrm{mAb}$ 's. In later studies by Heller et al. in which a more prolonged form of oxazolone colitis was studied, it was shown that the initial IL-4 response is rapidly superseded by an IL-13 response, and, in fact, the inflammation is also prevented by the administration of an IL-13 inhibitor, IL-13R $\alpha 2-\mathrm{Fc}$ (10). Further studies by these authors revealed that NK T (NKT) cells rather than conventional $\mathrm{CD}^{+}{ }^{+} \mathrm{T}$ cells mediate oxazolone colitis and, importantly, NKT cells were the source of the IL-13, since they produced IL-13 upon stimulation by $\alpha$-galactosylceramide ( $\alpha$-GalCer), a glycolipid antigen that is recognized by the invariant $\mathrm{T}$ cell receptor (TCR) usually associated with NKT cells $(11,12)$.

The resemblance of oxazolone colitis to UC at the histopathological level leads to the question of whether the mechanisms resulting in the murine model also underlie the human disease. In the present study, we addressed this question and found that involved tissue from UC patients contain increased numbers of nonclassical NKT cells (i.e., NKT cells that do not bear an invariant TCR) that produce markedly increased amounts of IL-13. In addition, we show that these cells are cytotoxic for epithelial cell targets and that the cytotoxicity is enhanced by IL-13. These data strongly suggest that UC is characterized by an atypical Th2 response having the potential of causing the epithelial cell damage that is the hallmark of the disease. 

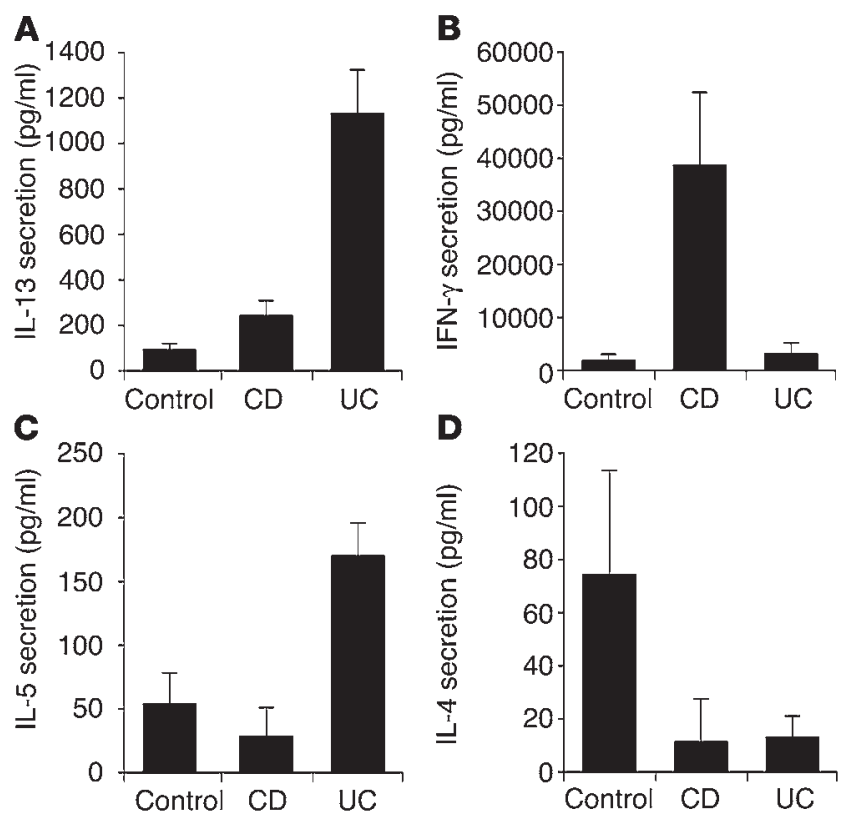

\section{Results}

IL-13 production in UC. In initial studies, we determined the capacity of lamina propria mononuclear cells (LPMCs) extracted from tissues obtained from patients with UC, CD, and control patients to produce IL-13, IL-4, IL-5, and IFN- $\gamma$ following stimulation in vitro with polyclonal stimuli, either anti-CD2/anti-CD28 or anti-CD3/antiCD28 (see Methods). As shown in Figure 1A (depicting stimulation with anti-CD2/anti-CD28), while LPMCs from both UC patients and CD produced greater amounts of IL-13 than control individuals, the increase exhibited by LPMCs from UC patients was far great-

\section{Figure 2}

Intracellular cytokine expression of CD161+ LPMC. (A) CD161 expression on unstimulated CD3 $3^{+}$LPMCs. LPMCs from control $(n=5)$, involved CD $(n=6)$, and involved UC $(n=8)$ tissues were stained with fluorochrome-labeled Ab's recognizing various surface antigens (CyChrome-labeled anti-CD3, PE-labeled anti-CD4, and FITC-labeled anti-CD161) and then were analyzed for the expression of $\mathrm{CD} 4^{+}$versus CD161+ cells on CD3-gated cells. (B) Intracellular IL-13 cytokine production by stimulated LPMCs. LPMCs from control $(n=5)$, involved $\mathrm{CD}(n=6)$, and involved UC $(n=8)$ tissues were cultured for 48 hours with anti-CD2/anti-CD28 with addition of monensin for the last 6 hours of culture. The resultant cells were stained with fluorochrome-labeled Ab's recognizing various surface antigens (CyChrome-labeled antiCD3, APC-labeled anti-CD8, and FITC-labeled anti-CD161) and then were fixed/permeabilized prior to intracellular cytokine staining with PE-labeled anti-IL-13. Cells were gated on CD3 and analyzed for the expression of CD8 ${ }^{-}\left(\mathrm{CD} 4^{+}\right)$cells and $\mathrm{CD} 161^{+}$cells versus IL-13+ cells. (C) Intracellular IFN- $\gamma$ cytokine production by stimulated LPMCs was the same as in B, except LPMCs were cultured for 6 hours with PMA and ionomycin with the addition of monensin for the last 5 hours of culture, and fixed/permeabilized cells were stained with APC-labeled anti-IFN- $\gamma$. (D) Intracellular IL-13 cytokine production by unstimulated LPMCs. LPMCs from involved CD $(n=4)$ and involved UC $(n=4)$ tissues were stained with fluorochrome-labeled $A b$ 's recognizing various surface antigens (CyChrome-labeled anti-CD3, APC-labeled anti-CD8, and FITC-labeled anti-CD161) and then fixed/permeabilized prior to intracellular cytokine staining with PE-labeled anti-IL-13. Cells were gated on CD3 and analyzed for the expression of $\mathrm{CD} 4^{+}$cells and CD161+ cells versus IL-13+ cells.

\section{Figure 1}

Cytokine secretion by control, CD, and UC LPMCs. (A) IL-13, (B) IFN- $\gamma$, (C) IL-5, and (D) IL-4 secretion by LPMCs obtained from control $(n=6)$, involved CD $(n=8)$, and involved UC $(n=12)$ tissues induced by antiCD2/anti-CD28. In all experiments error bars represent SEMs. Similar secretion patterns for all cytokine analyzed were found with anti-CD3/ anti-CD28 stimulation of LPMCs.

er than the one observed in LPMCs from CD patients (tenfold and two- to threefold respectively), and UC LPMCs produced three- to fourfold greater amounts of IL-13 than CD LPMCs, a highly significant difference $(P<0.01)$. Similarly, as previously demonstrated (8) and shown in Figure 1C (depicting stimulation with anti-CD2/ anti-CD28), LPMCs from UC patients also produced a significantly greater amount of IL-5 as compared with control or CD LPMCs $(P<0.01)$, and in this case, control and CD LPMCs produced the same low levels. As shown in Figure 1D and in previous studies, however, LPMC production of IL-4, another Th2 cytokine, was at least fivefold lower in cells obtained from either UC or CD patients as compared with cells obtained from controls and did not differ significantly from one another $(8,13)$. The above changes in cytokine production were most likely limited to areas of inflammation because in the three cases studied in which involved and uninvolved tissues were examined, cells from the UC-uninvolved tissue manifested no increase in IL-13 secretion compared with controls $(108 \pm 10$ vs. $87 \pm 33 \mathrm{pg} / \mathrm{ml}$ ). Finally, with respect to secretion of the Th1 cytokine, IFN- $\gamma$, as shown in Figure 1B, LPMCs from CD patients produced greater than tenfold higher amounts of IFN- $\gamma$ than LPMC from UC patients $(P<0.01)$; the latter produced amounts of IFN- $\gamma$

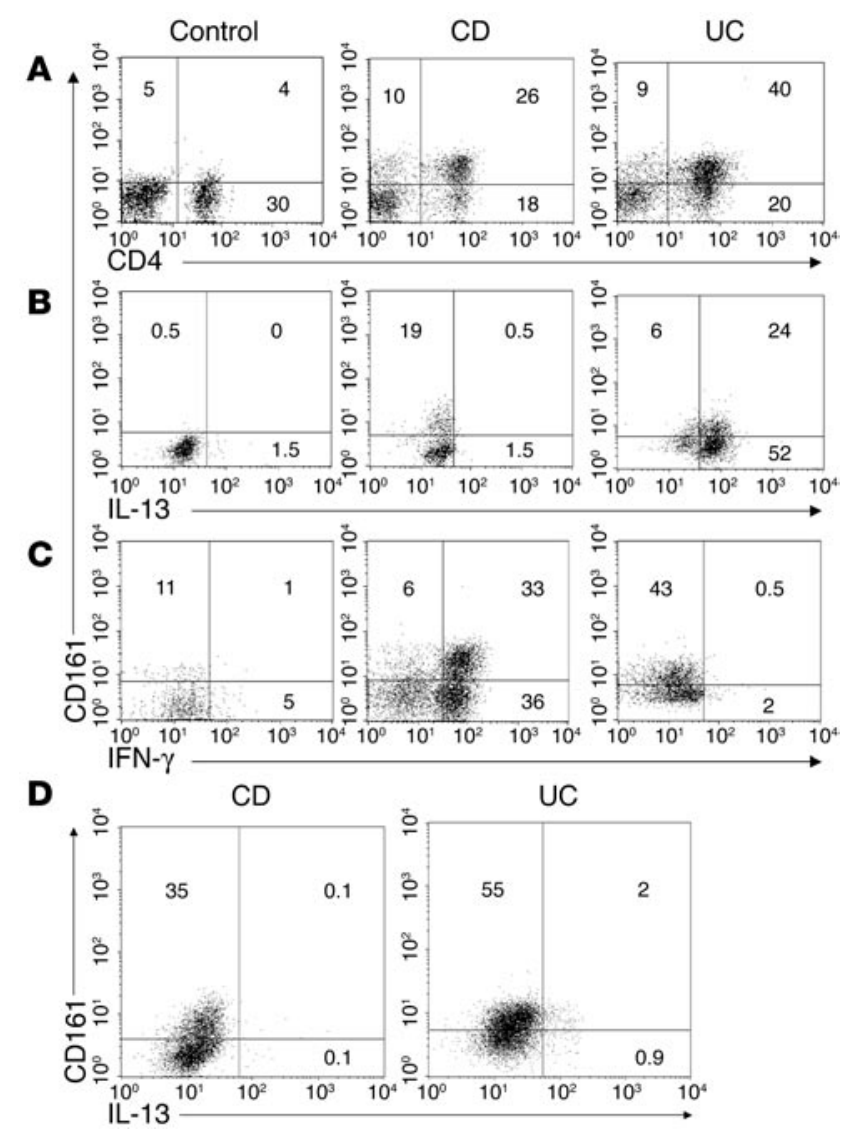



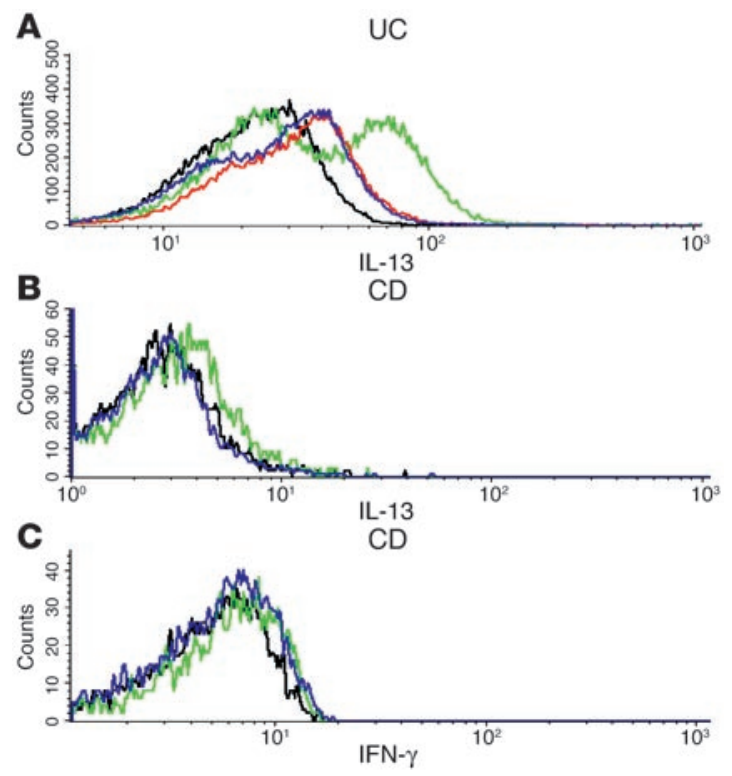

\section{Figure 3}

Stimulation of cytokine production in LPMCs by EBV-transformed B cells expressing surface CD1d. (A) LPMCs isolated from involved UC lamina propria were cultured for 48 hours with PMA/IL-2 (black line) (see Methods) in the presence or absence of EBV-transformed $B$ cells transfected with CD1d (721 cells) (green line) with or without anti-CD1d mAb (51.1.3) (red line) or untransfected 721 (WT) B cells (blue line). (B and C) LPMCs isolated from involved CD lamina propria were treated as in $\mathbf{A}$. In all cultures monensin was added 6 hours prior to cell harvest. Cells were initially surface stained with fluorochromelabeled Ab's (CyChrome-labeled anti-CD3 and APC- or PE-labeled anti-CD8) and then fixed/permeabilized prior to intracellular staining with PE-labeled anti-IL-13 or APC-labeled anti-IFN- $\gamma$. CD3-gated cells were then analyzed for $\mathrm{CD} 4^{+}$versus IL- $13^{+}$or IFN- $\gamma$ cells. Histogram shown is representative of three for each patient population.

equal to that of LPMCs from controls. As an additional control for these studies we also conducted kinetic time course studies of both IL-13 and IL-5 secretion and found that peak secretion of both these cytokines occurred at 48-72 hours. We also found that the difference between UC and CD cells was seen throughout the 72-hour time period (data not shown). Taken together, these results demonstrate that LPMC production of IL-13 and IL-5 but not IL-4 is high in UC as compared with CD patients, and production of IFN- $\gamma$ is high in CD as compared with UC patients.

The proportion of cells in UC/CD LPMCs bearing an NKT cell marker (CD161) and secreting IL-13 and/or IFN- $\gamma$. We next sought to determine the cellular source of the increased IL-13 secretion noted above. Given the fact that in oxazolone colitis, an experimental model of colitis resembling UC, NKT cells were the source of the IL-13, we focused on the possibility that a similar cell was responsible for the high IL-13 production in UC. In initial studies along these lines we sought to determine the level of NKT cells in LPMC populations extracted from tissues of $\mathrm{UC}, \mathrm{CD}$, and control patients using the CD161 (NK1.1) marker. We recognized, however, that this approach would be limited by the fact that even though CD161 is the widely used marker of human NKT cells this marker is also found on activated $\mathrm{CD} 4^{+}$cells and thus could yield false-positive results in studies of LPMC populations that may contain large numbers of activated cells $(14,15)$. As shown in the representative dot plot depicted in
Figure $2 \mathrm{~A}$, an increased $\mathrm{CD} 4^{+} \mathrm{CD} 161^{+}$expression was observed in unstimulated LPMCs from both UC and CD patients and were not significantly different in these groups; however, these levels were significantly higher than those in control LPMCs $(P<0.01)$. The range of expression of $\mathrm{CD} 4{ }^{+} \mathrm{CD} 161^{+} \mathrm{T}$ cells for control patients were $1.14-6 \%$ (mean 3.2\%), for CD patients were $18.71-58.1 \%$ (mean $31.4 \%$ ), and for UC patients were 16.2-61.3\% (mean 33.8\%).

While these studies did not identify a quantitative difference in the number of CD161+ $\mathrm{T}$ cells in UC patients as compared with CD patients, presumably because of the ambiguous significance of the CD161 marker referred to above, it was still possible that this population of cells in the two patient groups differed qualitatively. To investigate this possibility we next determined if the CD $161^{+}$ cell population harbored a subpopulation of cells producing IL-13. Accordingly, we stimulated LPMCs from the various patient groups with anti-CD2/anti-CD28 and then subjected the cells to fourcolor flow-cytometric analysis using staining reagents recognizing CD3, CD8, CD161, and intracellular IL-13. As shown in a representative dot plot depicted in Figure 2B (derived from the same individuals studied in Figure 2A), UC LPMCs contained a greater than 30 -fold higher percentage of $\mathrm{CD} 4^{+} \mathrm{CD} 161^{+} \mathrm{T}$ cells producing IL-13 than CD LPMCs. The range of expression of CD $4^{+} \mathrm{CD} 161^{+} \mathrm{T}$ cells secreting IL-13 for control patients was $0-1.02 \%$ (mean $0.5 \%$ ), for $\mathrm{CD}$ patients was $0.01-1.92 \%$ (mean $0.66 \%$ ), and for UC patients was $7.2-51 \%$ (mean 26.6\%). As shown in Figure 2C (again derived from these same individuals), however, CD LPMCs contained a greater than 30 -fold higher percentage of $\mathrm{CD} 4{ }^{+} \mathrm{CD} 161^{+}$cells producing IFN- $\gamma$ than did UC LPMCs, and UC production of IFN- $\gamma$ was not higher than that observed in control LPMCs. The range of expression of $\mathrm{CD}^{+} \mathrm{CD} 161^{+} \mathrm{T}$ cells secreting IFN- $\gamma$ for control patients was $0.4-3 \%$ (mean $1.6 \%$ ), for CD patients was $10-56.3 \%$ (mean $35.2 \%$ ), for UC patients was $0.1-2.68 \%$ (mean $1.2 \%$ ). These results are consistent with the mitogen responses shown in Figure 1 and lead to the conclusion that CD $161^{+}$cells in UC and CD LPMCs exhibit markedly different cytokine-secretion profiles, whereas UC is associated with IL-13 secretion by a CD $161^{+}$cell, CD is not.

It should be noted that the above differences between UC and CD could be influenced by an additional limitation of the use of the CD161 marker, namely that its expression is downregulated by prolonged in vitro cell stimulation and activation in UC and CD cell populations (15). One would anticipate that this effect would affect both patient cell populations equally, however. This expectation is, in fact, noted in Figure 2B, which demonstrates that there

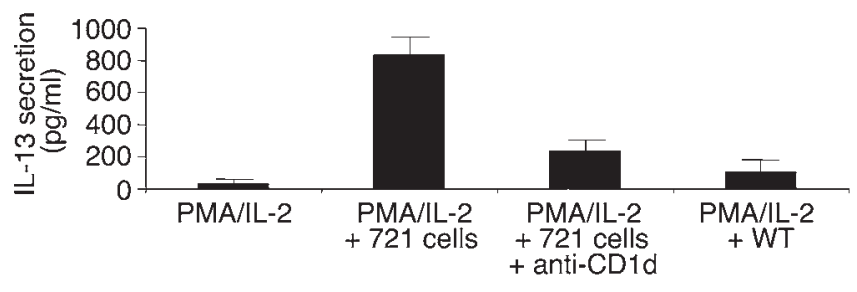

\section{Figure 4}

IL-13 secretion of UC LPMCs stimulated with EBV-transformed B cells transfected with CD1d. LPMCs extracted from involved UC tissue were cultured for 48 hours with PMA/IL-2 (see Methods) in the presence or absence of EBV-transformed B cells transfected with CD1d (721 cells) or untransfected 721 WT cells in the presence and absence of antiCD1d mAb (51.1.3). Culture supernatants were assayed by ELISA for the secretion of IL-13. Error bars represent SEMs. 


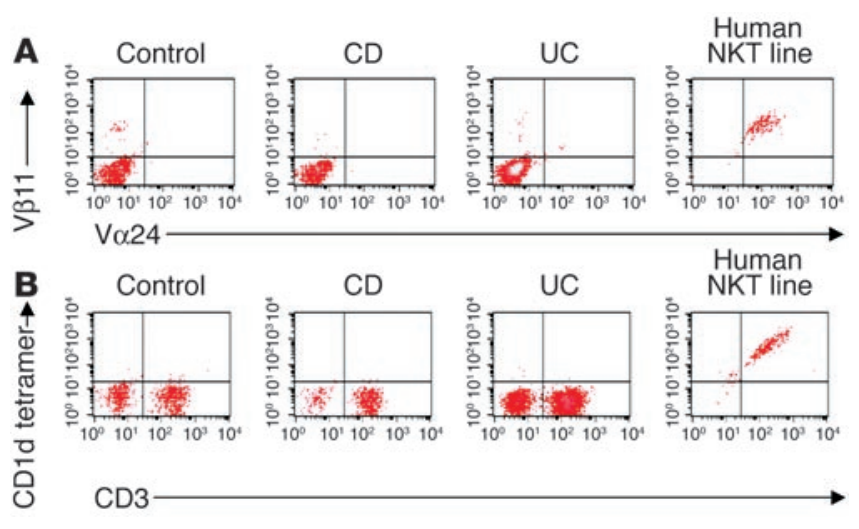

is roughly equal downregulation of the expression of CD $4^{+} \mathrm{CD} 161^{+}$ in LPMCs from UC and CD patients after stimulation. In further studies to more directly overcome this limitation, we determined the expression of intracellular IL-13 in CD and UC CD4 ${ }^{+} \mathrm{CD} 161^{+}$ cells prior to cell activation. As shown in the representative dot blot depicted in Figure 2D $(n=4)$, we observed fewer UC cells expressing IL-13 under these conditions because optimal expression requires cell activation; nevertheless, about 20-fold more IL-13expressing cells were seen in UC cells as compared with CD cells, and approximately $75 \%$ of the IL-13-expressing cells were CD161 positive. In a second study along these lines, we depleted UC LPMCs of CD $161^{+}$cells prior to cell activation (with anti-CD2/ anti-CD28) using anti-CD161-coated Miltenyi Biotec beads (see Methods) to determine if IL-13-producing cells initially reside in a CD $161^{+}$cell population. Indeed, if UC LPMC populations are subjected to such depletion, the amount of IL-13 production falls from $832 \pm 63 \mathrm{pg} / \mathrm{ml}$ to $57 \pm 6 \mathrm{pg} / \mathrm{ml}(n=3)$. In this study we also determined if IL- 5 production is also affected by CD161 depletion prior to cell activation and found that IL-5 levels decreased from $133 \pm 9 \mathrm{pg} / \mathrm{ml}$ to $22 \pm 12 \mathrm{pg} / \mathrm{ml}(n=3)$. This study thus offered evidence that IL-5 was also being produced by CD $161^{+} \mathrm{T}$ cells.

Stimulation of UC/CD LPMC with cells bearing high levels of CD1d. If, indeed, the $\mathrm{CD} 4{ }^{+} \mathrm{CD} 161^{+}$cells producing IL-13 in UC are NKT cells, as implied above, they should exhibit a prime characteristic of NKT cells, namely that their responses are CD1d restricted $(16,17)$. To determine if this is the case, we took advantage of the fact that all CD1d-restricted T cells are stimulated by APCs that bear high levels of surface CD1d under certain stimulation conditions $(16,18,19$, $20)$. We thus cocultured LPMCs from the various patient groups with an EBV-transformed B cell line that expressed high levels of CD1d as a result of transfection with a CD1d construct (the 721.221 cell line, referred to here as 721 cells) and then subjected the cells to flowcytometric analysis to determine IL-13/IFN- $\gamma$ secretion as described above. It should be noted that such cocultures were carried out in the presence of subactivating concentrations of PMA/IL-2, which has previously been shown to be required for such stimulation $(18,21)$. As shown in Figure 3, stimulation of LPMCs from UC patients with 721 cells transfected with CD1d resulted in a fourfold increase in the number of IL-13-secreting cells as compared with stimulation with PMA/IL-2 alone or cells cocultured with untransfected 721 cells (721 WT cells). In contrast, UC LPMCs stimulated in a similar fashion with 721 cells transfected with CD1d resulted in little increase in IFN- $\gamma$ secretion (data not shown). Moreover, coculture of LPMCs from UC patients with 721 cells transfected with CD1d in the presence of a $\mathrm{mAb}$ (51.1.3) that inhibits CD1d interaction with CD1d-restricted

\section{Figure 5}

Expression of invariant chain TCR in LPMC $\mathrm{CD}^{+}$subpopulations. (A) LPMCs isolated from control, involved CD and UC tissues, as well as a control NKT cell line (see Methods), were stained with various fluorochrome-labeled Ab's (CyChrome-labeled anti-CD3, FITC-labeled anti-V $\alpha 24$, and PE-labeled anti-V $\beta 11$ ). The resultant cells were gated on CD3 and analyzed for the expression of $V_{\alpha} 24$ versus $V \beta 11$. (B) LPMCs isolated from control, involved CD and UC tissues, as well as a control NKT cell line, were stained with FITC-labeled anti-CD3 and PE-labeled $\alpha$-GalCer-loaded CD1d tetramer. The resultant cells were then analyzed for surface expression of CD3 versus $\alpha$-GalCer-loaded tetramer. The dot plots in both $\mathbf{A}$ and $\mathbf{B}$ are representative of results from three experiments.

$\mathrm{T}$ cells resulted in a significant drop in the number of UC LPMCs producing IL-13, so that in the presence of the Ab the number did not differ from that of cells producing IL-13 cocultured with 721 cells not transfected with CD1d. In contrast, as also shown in Figure 3, LPMCs from CD patients did not secrete increased amounts of IL-13 or IFN- $\gamma$ when stimulated with 721 cells transfected with CD1d. The absence of a CD1d-reactive cell population in CD LPMCs suggests that the increased CD161 cell population observed in Figure 2 secreting large amounts of IFN- $\gamma$ represents a "conventional" activated $\mathrm{CD} 4^{+}$cell population that is not CD1d restricted. It should also be noted that stimulation of UC LPMCs with cells bearing substantially less surface CD1d (C1R B cells transfected with CD1d) (21) led to reduced but still measurable IL-13 in three other patients (data not shown), indicating that optimal IL-13 production requires stimulation with cells expressing high levels of CD1d.
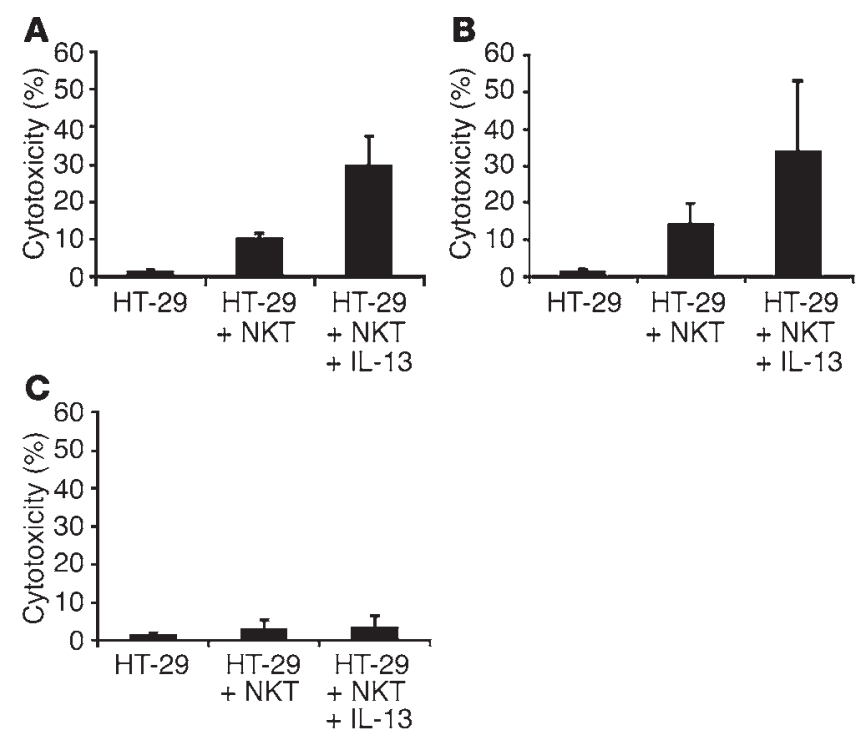

\section{Figure 6}

Cytotoxicity of NKT cells. In these studies HT-29 epithelial cells were prestimulated with LPS (see Methods) to induce increased expression of CD1d (data not shown). (A) Invariant NKT cells were cultured with target HT-29 cells in the presence or absence of IL-13 Ab (see Methods), and the percentage of cytotoxicity was measured by the CytoTox 96 System ( $n=4$ experiments). Error bars represent SEMs. (B) Purified UC lamina propria (LP) $\mathrm{CD} 4^{+} \mathrm{CD} 161^{+} \mathrm{T}$ cells were cultured as in $\mathbf{A}$. (C) Purified CD LP CD4+CD161+ T cells were cultured as in A. For both B and $\mathbf{C}, n=2$ experiments. Error bars represent SDs. 
Finally, the studies of UC LPMC IL-13 production resulting from stimulation by 721 cells measured by flow-cytometric analysis were fully corroborated by studies of IL-13 secretion measured by ELISA. Thus, as shown in Figure 4, UC LPMCs stimulated with 721 cells transfected with CD1d produce a significantly increased amount of IL-13 as compared with cells stimulated with PMA/IL-2 alone $(P<0.01)$. In addition, this increased IL-13 secretion was significantly reduced in the presence of anti-CD1d mAb (51.1.3) $(P<0.01)$. The addition of control IgG Ab instead of anti-CD1d mAb led to no measurable change in the increased IL-13 secretion (data not shown). In contrast, CD LPMC showed no increase in IL-13 or IFN- $\gamma$ secretion (above the baseline secretion levels achieved with PMA/IL-2) when stimulated with transfected 721 cells (data not shown).

Lack of cells in UC LPMCs bearing an invariant TCR. In previous studies of both mouse and human NKT cells it has been noted that a major subpopulation of NKT cells bear an "invariant" TCR identified by a particular $V \alpha$ and $J \alpha$ chain and a capacity to bind a specific tetramer loaded with $\alpha$-GalCer, a glycolipid presented by APCs expressing CD1d and shown previously to react with invariant TCR NKT cells $(16,22,23,24)$. In addition, it has been shown that invariant TCR NKT cells exhibit the ability to proliferate or produce cytokine when stimulated by $\alpha$-GalCer $(11,24)$. Indeed, NKT cells with an invariant TCR were shown to be the IL-13-producing cell in oxazolone colitis. On this basis we determined if the NKT cell identified in UC also expresses the invariant TCR associated with most NKT cells.

Accordingly, we first stained unstimulated LPMCs from the various patient populations with mAb's specific for $V \alpha 24$ and $V \beta 11$, since these specificities have previously been shown to characterize human invariant NKT cells $(19,25)$. As shown in Figure 5A, the number of cells in both UC and CD LPMC populations expressing these specificities were quite low (0.1-0.4\%) and did not differ either from each other or from the percentage of these cells in control LPMCs. In further studies in this vein, we also stained $\mathrm{CD}^{+}$LPMCs with an $\alpha$-GalCer-loaded tetramer capable of recognizing human invariant NKT cells (see Methods). As shown in Figure 5B, only a very small population of LPMCs from both UC and $\mathrm{CD}$ patients $(0.1-0.4 \%)$ exhibited positive staining with the $\alpha$-GalCer-loaded tetramer. In contrast, as shown in the control study in Figure 5, 99\% of the $\mathrm{CD}^{+}$cells comprising a known invariant NKT cell line were tetramer positive.

In the final and perhaps the most definitive study seeking the presence of invariant NKT cells in UC, we stimulated LPMC populations from the various patient groups with $\alpha$-GalCer. LPMCs from both UC and CD patients stimulated in this way produced only negligible amounts of IL-13 $(2.5 \pm 1 \mathrm{pg} / \mathrm{ml}$ and $0 \mathrm{pg} / \mathrm{ml}$, or undetectable amounts) in UC and CD, respectively. Taken together, these various studies establish that invariant TCR NKT cells are not increased in UC, and these cells are not the source of the IL-13 produced by $\mathrm{CD} 4^{+} \mathrm{CD} 161^{+} \mathrm{CD} 1 \mathrm{~d}$-restricted $\mathrm{T}$ cells in this disease.

NKT cells in UC exhibit IL-13-augmented cytotoxicity for epithelial target cells. In an attempt to determine if the NKT cells identified in UC patients have the potential to mediate tissue injury in this disease as previously shown in various animal models (26-28), we conducted studies to measure their ability to mediate cytotoxic function. In initial studies we determined the capacity of human NKT cell lines bearing the invariant TCR associated with human NK cells, prepared as described by Exley et al. (i.e., classical NKT cells) (21) to mediate cytotoxicity for LPS-treated HT-29 epithelial cells, a highly differentiated human epithelial cell line that expressed CD1d following LPS treatment (data not shown). As shown in Figure 6A, the NKT cell lines exhibited modest cytotoxicity for HT-29 cells (10:1 effector-to-target ratio [E/T]), which was enhanced by the presence of IL-13 $(P<0.01)$ but was not increased further by the addition of TNF- $\alpha$, another inflammatory cytokine that may be increased in UC (data not shown). The addition of anti-CD1d Ab to the culture led to an approximate $70 \%$ decrease in the enhancement induced by IL-13 (data not shown). In further studies focusing on NKT cells in UC and CD patients we conducted cytotoxicity studies with purified $\mathrm{CD} 4^{+} \mathrm{CD} 161^{+}$cells again using LPS-treated HT-29 cells as targets. As shown in Figure 6B, UC CD $4^{+} \mathrm{CD} 161^{+}$ cells exhibited modest cytotoxicity for HT-29 targets that was also enhanced by the presence of IL-13; again the presence of anti-CD1d $\mathrm{Ab}$ in the culture led to a similar decrease in IL-13 enhancement as seen with the NKT cell line. In contrast, as shown in Figure 6C, a similar cell population isolated from CD patients exhibit a low baseline cytotoxicity and no significant enhancement by IL-13. We conclude from these studies that NKT cells in UC lamina propria do have the potential of acting as cytotoxic cells for epithelial cells.

\section{Discussion}

In the present study we found that LPMCs from involved tissue of UC patients contain $\mathrm{CD}^{+}$cells bearing an NKT cell marker (CD161) that, upon polyclonal activation, produces increased amounts of IL-13 (and IL-5) as compared with equivalent cells from control or CD tissue; in contrast, similar cells from CD patients produced increased amounts of IFN- $\gamma$. These findings were corroborated by four-color flow cytometry in which we showed that $\mathrm{CD}^{+} \mathrm{T}$ cells from UC patients bearing an NKT cell marker (CD161) express intracellular IL-13, whereas similar cells from CD patients express intracellular IFN- $\gamma$. Finally, we showed that UC LPMCs produce IL-13 when cocultured with a B cell line expressing high levels of surface CD1d (in the presence of PMA and IL-2), indicating that the IL-13 was being secreted by a CD1d-restricted cell. Collectively, these data strongly suggest that IL-13-producing, CD1d-restricted, NKT cells are uniquely associated with the UC but not the CD form of IBD.

The observation that UC is characterized by an increased Th2oriented immune response partially coincides with an earlier study in which it was shown that in intestinal biopsies IL-13 mRNA, as well as IL-4 and IL-10 mRNA, was increased significantly in a larger percentage of UC patients than in "noninflammatory" controls; however, these results were difficult to interpret because IL-13 and IL-10 mRNA was not increased compared with "inflammatory" controls (including CD patients) (29). In addition, our findings differed in that, in concert with prior studies, we found a decrease in IL-4 protein secretion rather than an increase in IL-4 mRNA (8, 13). We postulate that this decrease is due to the fact that an initial IL-4 response in UC is superseded by an IL-13 response, as it is in oxazolone colitis (10). It will be interesting in this regard to examine patients early after onset of UC to determine if the lesion is initially associated with IL-4.

The Th2 orientation of the UC lesion is also congruent with an earlier finding that mRNA encoding a recently discovered cytokine chain known as EBI3 was shown to be increased in UC tissues as compared with CD or control tissues $(30,31)$. Of interest, EB13-deficient mice manifest poor Th2 responses and are resistant to the development of oxazolone colitis (32). In addition, while such mice exhibit normal numbers of both naive and 
mature $\mathrm{CD}^{+}$and $\mathrm{CD}^{+}$cells, they have markedly reduced numbers of NKT cells. These studies thus suggest that an EBI3-associated cytokine (such as IL-27) may be necessary for the development of the Th2-cytokine-producing NKT cells that characterize both UC and oxazolone colitis.

NKT cells are defined by the fact that they are cells expressing both a TCR and NK receptors such as NK1.1 (CD161) (reviewed in ref. 16, 28). In addition, NKT cells recognize antigens (usually glycolipid antigens) in association with CD1d (CD1 in mice), a MHC class I-like molecule present on the surface of professional APCs (dendritic cells) as well as on nonprofessional APCs such as intestinal epithelial cells (IECs) (33). The TCRs used by classic NKT cells are invariant in that they use a particular $\mathrm{V} \alpha$ chain (in humans, $\mathrm{V} \alpha 24 / \mathrm{J} \alpha \mathrm{Q}$ ) usually associated with a particular $V \beta$ chain (in humans, V $\beta 11)(19,34)$. On this basis, the classical invariant NKT cells (iNKT cells) recognize a simple glycolipid, $\alpha$-GalCer, and can be identified with $\alpha$-GalCer-loaded tetramers $(16,23,24)$. Since glycolipids are also found among self-antigens or antigens in the mucosal microflora, NKT cells may be self-antigen-reactive cells. This probably explains the finding noted here that they react to cells expressing CD1d in the absence of exogenous antigen, presumably because under these circumstances a self-glycolipid is present within the CD1d groove.

Whereas invariant NK T cells comprise the majority of CD1drestricted NKT cells, the latter also include nonclassical NKT cells that express noninvariant (diverse) or, alternatively, semiinvariant TCRs that nevertheless react with antigens presented by CD1d (as do iNKT cells) $(16,23)$. The cell population producing IL-13 and associated with the UC lesion appear to belong to this category of nonclassical NKT cells since they do not express an invariant TCR as indicated by their inability to bind $\alpha$-GalCer tetramers and are stimulated by CD1d on the surface of CD1d-transfected EBV-transformed B cells (i.e., they are CD1d restricted) but not by $\alpha$-GalCer. Such reactivity to $\mathrm{CD} 1 \mathrm{~d}$ in the absence of a specific antigen required partial activation by PMA as well as high-level expression of CD1d on the surface of the stimulating cell, probably reflecting the fact the cells are being stimulated by a relatively low-affinity selfantigen expressed by B cells in association with CD1d, as discussed above. It is interesting to postulate that these non- or semi-invariant CD1-restricted (NKT) cells in patients with UC recognize glycolipids in the bacterial microflora of the gut that cross-react with glycolipid self-antigens. Studies to establish the nature of this antigen or class of antigens will be among the next important steps in the elucidation of UC.

NKT cells secrete both Th1 and Th2 cytokines very soon after activation and thus can be considered a component of the innate immune system that "conditions" subsequent adaptive immune responses $(15,35,36)$. In addition, NKT cells may also function as regulatory cells since there is evidence that these cells protect mice against the development of autoimmune diabetes, experimental allergic encephalitis, and intestinal inflammation, possible through the production of Th2 cytokines (37-40). More relevant to their role in UC, however, is evidence that NKT cells also act as effector cells that induce inflammatory disease. This is seen clearly not only in the oxazolone colitis model, but also in models of allergen-induced airway hyper-reactivity (41) and in concanavalin Ainduced (conA-induced) hepatitis, a model of autoimmune liver disease (42). In addition, it has recently been shown that non-iNKT (nonclassical) cells contribute to liver injury in a murine model of hepatitis B virus infection (43).

Another key finding in this study is that NKT cells, in general (in the form of iNKT cell lines), as well as the nonclassical NKT cells in UC tissues can act as cytotoxic cells for human epithelial target cells and that such cytotoxicity is enhanced by IL-13. This finding recalls the many studies of UC performed in the 1960s-1980s in which various investigators attempted to show that this disease is associated with (if not, in fact, due to) cells capable of causing epithelial cell cytotoxicity and thus the characteristic ulcerative lesions of UC (reviewed in ref. 44). These studies were ultimately unable to prove the existence of bona fide cytotoxic cells with ability to lyse epithelial cells in UC, but, of course, they were performed before the present knowledge of NKT cells and their requirements for CD1d-expressing targets and IL-13 was available. It should be noted in this context that the target cells used in these studies were LPS-stimulated human epithelial target cells that expressed increased levels of CD1d as a result of LPS stimulation and, indeed, cytotoxicity was blocked by the addition of anti-CD1d. On the basis of these cytotoxicity findings it is reasonable to postulate that the immunopathology of UC involves first the stimulation of NKT cells through antigen presented to these cells by CD1d-bearing DCs or epithelial cells. The NKT cells so stimulated then begin to produce IL-13, at which point they become capable of lysing epithelial cells, and this leads to epithelial cell loss, ulceration, and breaches in the IEC barrier. Further intensification of the inflammation then ensues due to entry of organisms into the lamina propria proper as well as a result of IL-13induced chemokine production (45) that leads to the influx of acute inflammatory cells. Evidence for this postulated series of events as a cause of UC are the aforementioned studies of conA-induced hepatitis in which it has been shown that NKT cells cause hepatocellular cytolysis $(26,42)$ and the studies of oxazolone colitis in which it was shown that the inflammation can be aborted by agents that block either NKT cell formation or function or IL-13 (10). Further work in which specific inhibitors of IL-13 and/or NKT cells are administered to patients will be necessary to prove this hypothesis, however.

\section{Methods}

Patient population. Colonic specimens obtained from 31 surgical patients admitted for bowel resection to the Brigham and Women's Hospital (Boston, Massachusetts, USA), Istituto Superiore di Sanità (Rome, Italy), or Case Western Reserve University Hospital (Cleveland, Ohio, USA) for UC, CD, as well as malignant noninflammatory diseases, were studied. Fifteen patients with UC undergoing colectomy were evaluated based on the criteria of Truelove (46). Disease activity was severe in all patients. Ten surgical patients with CD were studied, and according to criteria established by De Dombal et al. (47), disease activity was classified as moderate in two and severe in eight patients. All $\mathrm{CD}$ patients had colonic involvement only. Finally, six surgical patients for bowel resection for malignant, noninflammatory condition were also studied. In every case these patients had undergone colonic resection secondary to adenocarcinoma. Collection of all surgical specimens was approved by Institutional Review Boards of the respective institutions.

Preparation of LPMCs. LPMCs were isolated from freshly obtained surgical specimens from IBD and non-IBD control patients using 
a modified technique previously described $(8,48)$. The resultant LPMC preparations contained greater than $95 \%$ viable cells, as assessed by trypan blue exclusion. In some experiments LPMC populations were stained with anti-CD161/FITC and separated into $\mathrm{CD} 161^{+}$and $\mathrm{CD} 161^{-}$fractions by using anti-FITC-coated (BD PharMingen, San Diego, California, USA) magnetic beads (Miltenyi Biotec, Auburn, California, USA). The resultant fractions were greater than $92 \%$ pure after separation.

Assay of LPMC cytokine production. Culture of LPMCs was performed using complete medium as previously described (8). The culture supernatants were harvested and assayed for cytokine concentrations by ELISA. In some experiments, $10^{6}$ LPMCs were cultured in the presence of $100 \mathrm{ng} / \mathrm{ml}$ of $\alpha$-GalCer for 48 hours (Kirin Co., Gunma, Japan).

ELISA assays. Cytokine concentrations were determined by commercially available specific ELISA assays for IFN- $\gamma$ and IL-4 (Pierce Chemical Co., Rockford, Illinois, USA; R\&D Systems Inc., Minneapolis, Minnesota, USA). IL-13 secretion was determined by using duo-paired cytokine coating (purified rat IgG1 anti-human IL-13 clone JES105A2; BD PharMingen) and detection Ab (clone SY04 biotinylated goat anti-human IL-13; R\&D Systems Inc.) according to modifications of the manufacturer's PharMingen ELISA protocol. Samples were analyzed against the standard curve generated from known quantities of recombinant human IL-13 (R\&D Systems Inc.) on an Immulon 4 plate (BD PharMingen). ODs were measured on a Dynatech MR 5000 ELISA reader at a wavelength of $450 \mathrm{~nm}$ for IFN- $\gamma$ and IL-4 and at $405 \mathrm{nM}$ for IL-13 measurements.

Intracellular cytokine detection by flow cytometry. Analysis of cells for the presence of intracellular IFN- $\gamma$ was performed on LPMCs stimulated with PMA ( $5 \mathrm{ng} / \mathrm{ml}$; Sigma-Aldrich, St. Louis, Missouri, USA) and ionomycin (100 ng/ml; SigmaAldrich) for 6 hours in complete medium in the presence of monensin (GolgiStop; BD Pharmingen). PMA/ ionomycin was found to be the optimal stimulus for IFN- $\gamma$ intracellular cytokine secretion because it did not significantly affect CD161 surface expression (see results in Figure 2B). Stimulation with anti-CD2/CD28 achieved similar intracellular secretion of IFN- $\gamma$, although, in this case, downregulation of CD161 expression was observed. For analysis of IL-13 secretion, monensin was added to the last 6 hours of LPMC cultures stimulated with anti-CD2/anti-CD28 or anti-CD3/anti-CD28, since these polyclonal stimulus were found to be optimal for IL-13 secretion. After stimulation, the cells were washed and labeled with conjugated $\mathrm{mAb}$ against surface antigens (CD3, CD8, $\mathrm{CD} 161)$. To analyze $\mathrm{CD}^{+} \mathrm{T}$ cell population, an analysis gate on $\mathrm{CD}^{+}{ }^{+} \mathrm{CD} 8^{-}$cells was established as significant down modulation of the CD4 occurs after stimulation. Cells were then fixed and permeabilized (Cytofix/Cytoperm; BD Pharmingen) for 20 minutes, washed with WashPerm (BD PharMingen), and labeled with cytokine-specific (APC-labeled, anti-IFN- $\gamma$ - and phycoerythrinlabeled [PE-labeled] anti-IL-13; BD PharMingen) conjugated $\mathrm{mAb}$ 's or isotype-matched Ig controls.

Flow-cytometric analysis. LPMCs were obtained from various patient populations as described. Cells were then washed three times with PBS and then stained with various fluorochromes using standard methods provided by the manufacturer (BD Pharmingen). Ab's used for surface-staining PE-labeled antiCD4, APC-labeled anti-CD8, PE-labeled anti-CD8, and FITClabeled anti-CD161 were purchased from BD PharMingen. FITC- labeled anti-V $\alpha 24$ and PE-labeled anti-V $\beta 11$ were purchased from Immunotech (Westbrook, Maine, USA). LPMCs were then analyzed by three- or four-color flow-cytometric analysis using FACSort and CellQuest software (Becton Dickinson Biosciences, San Diego, California, USA). Staining with PE-labeled CD1d- $\alpha-$ GalCer tetramers was performed as previously described (22). It should be noted that the cells were incubated with fluorochrome-labeled anti-CD3, V $\alpha 24$, and $V \beta 11$ for 30 minutes at $4{ }^{\circ} \mathrm{C}$, washed once with PBS staining buffer, and then reincubated with PE-labeled CD1d- $\alpha$-GalCer tetramer for 30 minutes at room temperature. Finally, the cells were washed twice with PBS staining buffer and analyzed by flow cytometry as described above (22). CD1d- $\alpha$-GalCer tetramers were prepared in the lab of R. Blumberg using materials kindly donated by Mitch Kronenberg (La Jolla Institute, University of California San Diego, La Jolla, California, USA). In some experiments a highly purified iNKT cell line was used as a control. This line was derived from peripheral blood cells obtained from a healthy donor that had been purified by a single-step sorting technique using an antiinvariant TCR $\mathrm{mAb}$ (6B11) specific for the complementary determining region 3 (CDR3) of the V $\alpha 24 / J \alpha Q$ TCR. The sorted cells were then expanded and maintained in the presence of anti-CD3 (OKT3) $(10 \mu \mathrm{g} / \mathrm{ml})$ and IL-2 $(100 \mathrm{U} / \mathrm{ml})(21)$.

Assay of NKT cell function with CD1d-transfected EBV-transformed $B$ cells. Assay of NKT cell function following stimulation by CD1d-transfected, EBV-transformed B cells was performed as described previously $(18,21)$. In brief, $5 \times 10^{5}$ LPMCs from each patient population were cultured with equal numbers of mitomycin-pretreated CD1d-transfected cells (721.722 B cells) or identical nontransfected cells for 48 hours. A stable CD1dtransfected 721.221-CD1d cell line was generated by transfecting the 721.221 cell line by electroporation with the pSR $\alpha$-neo vector containing the human CD1d cDNA as previously described (49). Transfected cells were selected by G418 sulfate resistance and screened by flow cytometry for CD1d expression. In some assays $30 \mu \mathrm{g} / \mathrm{ml}$ of 51.1 .3 -blocking CD1d mAb was added to the cell cultures. At the end of the culture period, cytokine measurements for intracellular or ELISA-secreted protein were performed as described above for IL-13 and IFN- $\gamma$.

Cytotoxicity assay. $\mathrm{CD}^{+} \mathrm{T}$ cells were obtained by the CD4 Multisort Kit (Miltenyi Biotec) and then selected for CD161 ${ }^{+} \mathrm{T}$ cells. NKT cells were maintained in IL-2 $(50 \mathrm{U} / \mathrm{ml}$; R\&D Systems Inc.) prior to culture. The iNKT cell line, purified UC and CD CD $161^{+} \mathrm{T}$ cells, were activated with either IL-13 $(20 \mathrm{ng} / \mathrm{nl}$; R\&D Systems Inc.) and/or TNF- $\alpha$ (20 ng/ml; R\&D Systems Inc.). The cells were then harvested and seeded with target HT-29 epithelial cells (American Type Culture Collection, Rockville, Maryland, USA) at the indicated $\mathrm{E} / \mathrm{T}$ ratio as previously described (50). In all studies, target cells were preincubated with LPS (SigmaAldrich) for 48 hours at a concentration of $1 \mu \mathrm{g} / \mathrm{ml}$. In some assays $30 \mu \mathrm{g} / \mathrm{ml}$ of 51.1 .3 blocking CD1d mAb was added to the cell cultures. Cytotoxicity activity was assayed using the CytoTox 96 System (Promega Corp., Madison, Wisconsin, USA) according to the manufacturer's specifications.

Statistical analysis. Statistical differences were assessed using the Student's $t$ test as indicated.

Note added in proof. Since submission and acceptance of this article, we note a recent publication by Jahng et al. (51) showing that experimental autoimmune encephalitis can be due to CD1drestricted NKT cells bearing a noninvariant TCR. 


\section{Acknowledgments}

The authors would like to thank Claudio Fiocchi for his extremely helpful technical support of this project. The authors would also like to thank Sara Kaul for her editorial assistance. R.S. Blumberg was supported by NIH DK-44319, 51362, and 53056 and the Harvard Digestive Diseases Center. M. Boirivant was partially supported by grant $1130 / \mathrm{RI}$ from the Italian Ministry of Health. This manuscript is dedicated to the memory of Abraham Fuss.

Received for publication August 19, 2003, and accepted in revised form March 23, 2004.

1. Sartor, R.B. 1995. Current concepts of the etiology and pathogenesis of ulcerative colitis and Crohn's disease. Gastroenterol. Clin. North Am. 24:475-507.

2. Strober, W., Fuss, I.J., and Blumberg, R.S. 2002. The immunology of mucosal models of inflammation. Annu. Rev. Immunol. 20:495-549.

3. Podolsky, D.K. 2002. Inflammatory bowel disease. N. Engl.J. Med. 347:417-429.

4. Fais, S.M., et al. 1991. Spontaneous release of interferon $\gamma$ by intestinal lamina propria lymphocytes in Crohn's disease: kinetics of in vitro response to interferon $\gamma$ inducers. Gut. 32:403-407.

5. Breese, E., Braeeger, P., Corrigan, C.J., Walker-Smith,J.A., and MacDonald, T.T. 1993. Interleukin-2 and interferon-gamma-secreting $\mathrm{T}$ cells in normal and diseased human intestinal mucosa. Immunology. 78:127-131.

6. Monteleone, G., et al. 1997. Interleukin 12 is expressed and actively released by Crohn's disease intestinal lamina propria mononuclear cells. Gastroenterology. 112:1169-1178.

7. Parronchi, P., et al. 1997. Type 1 T-helper cell predominance and IL-12 expression in the gut of patients with Crohn's disease. Am. J. Pathol. 150:823-832.

8. Fuss, I.J., et al. 1996. Disparate CD4+ lamina propria (LP) lymphokine secretion profiles in inflammatory bowel disease. Crohn's disease LP cells manifest increased secretion of IFN- $\gamma$, whereas ulcerative colitis LP cells manifest increased secretion of IL-5. J. Immunol. 157:1261-1270.

9. Boirivant, M., Fuss, I.J., Chu, A., and Strober, W. 1998. Oxazolone colitis, a murine model of Thelper cell type 2 colitis treatable with antibodies to interleukin 4 . J. Exp. Med. 188:1929-1939.

10. Heller, F., Fuss, I.J., Nieuwenhuis, E.E., Blumberg, R.S., and Strober, W. 2002. Oxazolone colitis, a Th2 colitis model resembling ulcerative colitis, is mediated by IL-13 producing NK-T cells. Immunity. 17:629-638.

11. Kawano, T., et al. 1997. CD1d-restricted and TCRmediated activation of vo14 NK T cells by galactosylceramide. Science. 278:1626-1629.

12. Spada, F.M., Koezuka, Y., and Porcelli, S.A. 1998. CD1d-restricted recognition of synthetic glycolipid antigens by human natural killer T cells. J. Exp. Med. 188:1529-1534.

13. West, G.A., Matsuura, T., Levine, A.D., Klein, J.S., and Fiocchi, C. 1996. Interleukin 4 in inflammatory bowel disease and mucosal immune reactivity. Gastroenterology. 110:1683-1695.

14. Lanier, L.L., Chang, C., and Phillips, J.H. 1994. Human NKR-P1A. A disulfide-linked homodimers of the C-type lectin superfamily expressed by a subset of NK and T lymphocytes. J. Immunol. 153:2417-2428.

15. Chen, H., and Paul, W.E. 1997. Cultured NK1.1+ $\mathrm{CD} 4+\mathrm{T}$ cells produce large amounts of IL-4 and IFN-gamma upon activation by anti-CD3 or CD1. J. Immunol. 159:2240-2249.

16. Kronenberg, M., and Gapin, L. 2002. The unconventional lifestyle of NK T cells. Nat. Rev. Immunol. 2:557-568.

17. Park, S.H., et al. 2001. The mouse CD1d-restricted repertoire is dominated by a few autoreactive $\mathrm{T}$ cell receptor families. J. Exp. Med. 193:893-904.

18. Exley, M., Furman, M., Porcelli, S., and Balk, S. 1998. CD161 (NKR-P1A) costimulation of CD1d-depen-
Address correspondence to: Ivan J. Fuss, Mucosal Immunity Section, National Institutes of Health, 10 Center Drive, Building 10, Room 11N238, Bethesda, Maryland 20892, USA. Phone: (301) 496-6810; Fax: (301) 402-2240; E-mail: ifuss@niaid.nih.gov.

Frank Heller's present address is: Department of Gastroenterology, Campus Benjamin Franklin, Charité-Universitätsmedizin Berlin, Berlin, Germany.

\section{Ivan J. Fuss and Frank Heller contributed equally to this work.}

dent activation of human $T$ cells expressing invariant Va24 JaQ T cell receptor a chains. J. Exp. Med. 188:867-876.

19. Lee, P.T., Benlagha, K., Teyton, L., and Bendelac, A. 2002. Distinct functional/lineages of human V (alpha) 24 natural killer T cells. J. Exp. Med. 195:637-641.

20. Capone, M., et al. 2003. Human invariant V alpha 24$\mathrm{J}$ alpha Q TCR supports the development of CD1ddependent NK1.1+ and NK1.1- T cells in transgenic mice. J. Immunol. 170:2390-2398.

21. Exley, M., Balk, S.P., and Wilson, B. 2004. Isolation and functional use of human NK T cells. In Current protocols of immunology. J.E. Coligan, A.M. Kruisbeek, D.H. Margulies, E.M. Shevach, and W. Strober, editors. John Wiley \& Sons. New York, New York, USA. Unit 14.11.

22. Matsuda, J.L., et al. 2000. Tracking the response of natural killer $\mathrm{T}$ cells to a glycolipid antigen using CD1d tetramers. J. Exp. Med. 192:741-754.

23. Gumperz, J.E., Miyake, S., Yamamura, T., and Brenner, M.B. 2002. Functionally distinct subsets of CD1d-restricted natural killer T cells revealed by CD1d tetramer staining. J. Exp. Med. 195:625-636.

24. Benlagha, K., Weiss, A., Beavis, A., Teyton, L., and Bendelac, A. 2000. In vivo identification of glycolipid antigen-specific $\mathrm{T}$ cells using fluorescent CD1d tetramers. J. Exp. Med. 191:1895-1903.

25. Porcelli, S., Yockey, C.E., Brenner, M.B., and Balk, S.P. 1993. Analysis of T cell receptor (TCR) expression by human peripheral blood CD4-CD $8-\alpha / \beta$ T cells demonstrates preferential use of several $V \beta$ genes and an invariant $\alpha$ chain. J. Exp. Med. 178:1-16.

26. Kaneko, Y., et al. 2000. Augmentation of V $\alpha 14$ NKT cell-mediated cytotoxicity by interleukin 4 in an autocrine mechanism resulting in the development of concanavalin A-induced hepatitis. J. Exp. Med. 191:105-114.

27. Kawano, T., et al. 1998. Natural killer-like nonspecific tumor lysis mediated by specific ligand-activated Va14 NNKT cells. Proc. Natl. Acad. Sci. U. S. A. 95:5690-5693.

28. Taniguchi, M., Harada, M., Kojo, S., Nakayama, T., and Wakao, H. 2003. The regulatory role of V $\alpha 14$ NKT cells in innate and acquired immune response. Annu. Rev. Immunol. 21:483-513.

29. Inoue, S., et al. 1999. Characterization of cytokine expression in the rectal mucosa of ulcerative colitis: correlation with disease activity. Am. J. Gastroenterol. 94:2441-2446.

30. Omata, F., Birkenbach, M., Matsuzaki, S., Christ, A.D., and Blumberg, R.S. 2001. The expression of IL-12 p40 and its homologue, Epstein-Barr virus-induced gene 3 , in inflammatory bowel disease. Inflamm. Bowel Dis. 7:215-220.

31. Christ, A.D., et al. 1998. An interleukin-12 related cytokine is upregulated in ulcerative colitis but not in Crohn's disease. Gastroenterology. 115:307-313.

32. Nieuwenhuis, E.S., et al. 2002. Disruption of Thelper 2 -immune responses in Epstein-Barr virus-induced gene 3-deficient mice. Proc. Natl. Acad. Sci. U. S. A. 99:16951-16956.

33. Van de Waal, Y., et al. Gastroenterology. Delineation of a Cd1d-restricted antigen presentation pathway associated with human and mouse intestinal epithelial cells. Gastroenterology. 124:1420-1431.
34. Takahashi, T., et al. 2000. Analysis of human V alpha 24 CD4+ NK T cells activated by alpha-glycosylceramide-pulsed monocyte-derived dendritic cells. J. Immunol. 164:4458-4464.

35. Yoshimoto, T., and Paul, W.E. 1994. CD4pos, NK1.1 pos $\mathrm{T}$ cells promptly produce interleukin 4 in response to in vivo challenge with anti-CD3. J. Exp. Med. 179:1285-1295.

36. Hayakawa, K., Lin, B.T., and Hardy, R.R. Murine thymic CD4+ T cell subsets: a subset (Th0) that secretes diverse cytokines and overexpresses the V beta $8 \mathrm{~T}$ cell receptor gene family. J. Exp. Med. 176:269-274.

37. Lehuen, A., et al. 1998. Overexpression of natural killerT cells protects V $\alpha 14-J \alpha 281$ transgenic nonobese diabetic mice against diabetes. J. Exp. Med. 188:1831-1839.

38. Wang, B., Geng, Y.B., and Wang, C.R. 2001. CD1restricted NK T cells protect nonobese diabetic mice from developing diabetes. J. Exp. Med. 194:313-320.

39. Singh, A.K., et al. 2001. Natural killer T-cell activation protects mice against experimental autoimmune encephalomyelitis. J. Exp. Med. 194:1801-1811.

40. Saubermann, L.J., et al. 2000. Activation of natural killer $\mathrm{T}$ cells by alpha-galactosylceramide in the presence of CD1d provides protection against colitis in mice. Gastroenterology. 119:119-128.

41. Akbari, O., et al. 2003. Essential roles of NK T cells producing IL-4 and IL-13 in the development of allergen-induced airway hyperreactivity. Nat. Med. 9:582-588.

42. Takeda, K., et al. 2000. Critical contribution of liver natural killer T cells to a murine model of hepatitis. Proc. Natl. Acad. Sci. U. S. A. 97:5498-5503.

43. Baron, J.L., et al. 2002. Activation of a nonclassical NK $T$ cell subset in a transgenic mouse model of hepatitis $B$ virus infection. Immunity. 16:583-594.

44. Strober, W., and James, S.P. 1986. The immunologic basis of inflammatory bowel disease. J. Clin. Immunol. 6:415-432.

45. Elias, J., Geun, C., Zheng, T., Shim, Y., and Zhu, Z. 2003. Interleukin 13 and leukotienes. An intersection of pathogenetic schema. Am. J. Respir. Cell Mol. Biol. 28:401-404.

46. Truelove, S.C., and Witts, L.F. 1955. Cortisone in ulcerative colitis: a final report on a therapeutic trial. Br. Med. J. 2:1041-1046.

47. De Dombal, F.T., Burton, I.L., Clamp, S.E., and Goligher, J.C. 1974. Short term course and prognosis of Crohn's disease. Gut. 15:435-439.

48. Bull, D.M., and Bookman, M.A. 1977. Isolation and functional characterization of human intestinal mucosal lymphoid cells. J. Clin. Invest. 59:966-974.

49. Balk, S.P., et al. 1991. Oligoclonal expansion and CD1 recognition by human intestinal intraepithelial lymphocytes. Science. 253:1411-1415.

50. Wunderlich, J., and Shearer, G. 2004. Assay for T cell function. Induction and measurement of cytotoxic T lymphocyte activity. In Current protocols of immunology. E. Coligan, A.M. Kruisbeek, D.H. Margulies, E.M. Shevach, and W. Strober, editors. John Wiley \& Sons. New York, New York, USA. Unit 3.11

51. Jahng, A., et al. 2004. Prevention of autoimmunity by targeting a distinct, noninvariant CD1d-reactive T cell population reactive to sulfatide. J. Exp. Med. 199:947-957. 